\title{
On the Logarithmic Regularity Conditions for the Variable Exponent Hardy Type Inequality
}

\author{
Aziz Harman and Mustafa Özgür Keleş \\ Faculty of Education, Dicle University, 21280 Diyarbakır, Turkey \\ Correspondence should be addressed to Aziz Harman; aharman@dicle.edu.tr
}

Received 11 November 2013; Revised 24 January 2014; Accepted 10 February 2014; Published 23 April 2014

Academic Editor: Yongsheng S. Han

Copyright (C) 2014 A. Harman and M. Ö. Keleş. This is an open access article distributed under the Creative Commons Attribution License, which permits unrestricted use, distribution, and reproduction in any medium, provided the original work is properly cited.

We discuss a logarithmic regularity condition in a neighborhood of the origin and infinity on the exponent functions $q(x) \geq p(x)$ and $\beta(x)$ for the variable exponent Hardy inequality $\left\|x^{\beta(\cdot)-1} \int_{0}^{x} f(t) d t\right\|_{L^{p(\cdot)}(0, l)} \leq C\left\|x^{\beta(\cdot)} f\right\|_{L^{p(\cdot)}(0, l)}$ to hold.

\section{Introduction}

There are several papers devoted to the variable exponent Hardy inequality:

$$
\left\|v^{1 / q(\cdot)} \int_{0}^{x} f(t) d t\right\|_{L^{q(\cdot)}(0, \infty)} \leq\left\|\omega^{1 / p(\cdot)} f(\cdot)\right\|_{L^{p(\cdot)}(0, \infty)} .
$$

Let $v, w: \mathbb{R} \rightarrow[0, \infty)$ be positive measurable functions and $p^{-}=\inf \{p(x): x \in \mathbb{R}\}>1, p^{+}=\sup \{p(x): x \in \mathbb{R}\}<$ $\infty$; then inequality (1) and (2) hold under certain conditions on the weight functions $v, \omega$ and the exponents $p(\cdot), q(\cdot)$. Two types of conditions arise here: a balance condition on the weights and a regularity condition on the exponents (see below). Necessary and sufficient conditions for the validity of general inequality (1) were found in [1] for the case of $p(x) \leq q(x)$, in [2] for cases $q(0) \geq p(0)$ and $q(\infty) \geq p(\infty)$, in [3] for cases $q(0)<p(0)$ and $q(\infty)<p(\infty)$, and in [4] for mixed cases $q(0) \geq p(0)$ and $q(\infty)<p(\infty)(q(0)<p(0)$ and $q(\infty) \geq p(\infty))$. Some special cases of (1) are studied in [5-10] too.

So, the inequality

$$
\left\|x^{\beta(\cdot)-1} H f(\cdot)\right\|_{L^{p(\cdot)}(0, \infty)} \leq C\left\|x^{\beta(\cdot)} f(\cdot)\right\|_{L^{p(\cdot)}(0, \infty)}
$$

is a particular case of (1) when $p(x)=q(x), v(x)=x^{\beta(x)-1}$ and $w(x)=x^{\beta(x)}$, where $H f(x)=\int_{0}^{x} f(t) d t$ is the Hardy operator. For the constant exponents $p, \beta$ this inequality holds if $\beta<1-(1 / p), p>1$ (see, e.g., [11]). Necessary and sufficient conditions on the $\beta(\cdot), p(\cdot)$ for inequality $(2)$ to hold are $\beta(0)<1-(1 / p(0))$ and $\beta(\infty)<1-(1 / p(\infty))$ if the exponents $p, q$ are continuous near the origin and infinity such that the conditions $p, \beta \in \Lambda_{0} \cap \Lambda_{\infty}$ are satisfied (see, e.g., $[5-7,9]$ ). In [8] (see also [10]) it was proved that the condition $p, \beta \in \Lambda_{1}$ is necessary for inequality (2) to hold if one of these exponents is a constant. Also, it was proved in [10] that the condition $\Lambda_{1}$ is sufficient for inequality (2) to hold on bounded interval $(0, l)$ if a constant $A_{1}$ in the condition $\Lambda_{1}$ for the $p$ satisfies $A_{1}<p(0)(p(0)-1)$ and the $\beta$ is zero. The condition $\Lambda_{1}$ is weaker then $\Lambda_{0}$. Also the function $p(x)=C / \sqrt{\ln (1 / x)}$ satisfies the condition $\Lambda_{1}$ but does not satisfy $\Lambda_{0}$.

In this note, we will focus on the results of sufficiency and necessity of regularity conditions $\Lambda_{0}, \Lambda_{\infty}$, and $\Lambda_{1}$ below for inequality (2) to hold.

The space of functions $L^{p(\cdot)}(0, \infty)$ is introduced as the class of measurable functions $f(x)$ in $(0, \infty)$, which have a finite $I_{p}(f):=\int_{0}^{\infty}|f(x)|^{p(x)} d x$ modular. A norm in $L^{p(\cdot)}(0, \infty)$ is given in the form

$$
\|f\|_{L^{p(\cdot)}(0, \infty)}=\inf \left\{\lambda>0, I_{p}\left(\frac{f}{\lambda}\right) \leq 1\right\} .
$$

As to the basic properties of spaces $L^{p(\cdot)}$, we refer to [12]. 


\section{Main Results}

We will state some sufficiency and necessity assertions concerning inequality (2). Along the way, it will be given a proof for two elementary estimates that we had used.

Let us introduce the following classes of measurable functions. We say, $s(x):(0, \infty) \rightarrow \mathbb{R}$ is in the class $\Lambda_{0}$ if

$$
\Lambda_{0}:=\limsup _{x \rightarrow 0}|s(x)-s(0)| \ln \frac{1}{|x|}<\infty,
$$

is in the class $\Lambda_{\infty}$ if

$$
\Lambda_{\infty}:=\limsup _{x \rightarrow \infty}|s(x)-s(\infty)| \ln |x|<\infty,
$$

and is in the class $\Lambda_{1}$ if

$$
\Lambda_{1}:=\limsup _{x \rightarrow 0}\left|s(x)-s\left(\frac{x}{2}\right)\right| \ln \frac{1}{|x|}<\infty .
$$

Theorem 1 (see $[8])$. Suppose $\beta \in \mathbb{R}$ and $p:(0, l) \rightarrow[1, \infty)$ is an increasing function on $(0, \epsilon)$ such that $p(x)$ is continuous at $x=0$ and $\beta<1-(1 / p(0)), p^{-}>1$; then for the inequality

$$
\left\|x^{\beta(x)-1} H f\right\|_{L^{p(\cdot)}(0, l)} \leq C\left\|x^{\beta(x)} f\right\|_{L^{p(\cdot)}(0, l)}
$$

to hold it is necessary that $p(\cdot) \in \Lambda_{1}$.

Theorem 2 (see [8]). Suppose $p \in \mathbb{R}$ and $\beta:(0, l) \rightarrow \mathbb{R}$ is a decreasing function on $(0, \epsilon)$ such that $\beta(x)$ is continuous at $x=0$ and $\beta(0)<1-(1 / p), p^{-}>1$; then for the inequality

$$
\left\|x^{\beta(\cdot)-1} H f\right\|_{L^{p(\cdot)}(0, l)} \leq C\left\|x^{\beta(\cdot)} f\right\|_{L^{p(\cdot)}(0, l)}
$$

to hold it is necessary that $\beta(\cdot) \in \Lambda_{1}$.

Theorem 3. Suppose $p, \beta:(0, \infty) \rightarrow \mathbb{R}$ is measurable functions such that $1<\beta^{-}, \beta^{+}<\infty, 1<p^{-}, p^{+}<\infty$; then for the inequality

$$
\left\|x^{\beta(\cdot)-1} H f(\cdot)\right\|_{L^{p(\cdot)}(0, \infty)} \leq C\left\|x^{\beta(\cdot)} f(\cdot)\right\|_{L^{p(\cdot)}(0, \infty)}
$$

to hold it is sufficient that $\beta, p \in \Lambda_{0} \cap \Lambda_{\infty}$, whenever $\beta(0)<$ $1-(1 / p(0))$ and $\beta(\infty)<1-(1 / p(\infty))$.

Theorem 4. Let $\beta \in \mathbb{R}, p:(0, \infty) \rightarrow[1, \infty)$ and let $p^{-}>$ 1. There exists a sequence $f_{n}$ and a function $p$, satisfying the conditions $\beta<1-(1 / p(0)), \beta<1-(1 / p(\infty))$ and

$$
\lim _{n \rightarrow \infty}\left|p\left(\delta_{n}\right)-p(0)\right| \ln \frac{1}{\delta_{n}}=\infty: \delta_{n}=4^{-n}
$$

or

$$
\lim _{n \rightarrow \infty}\left|\beta\left(\mu_{n}\right)-\beta_{\infty}\right| \ln \mu_{n}=\infty: \mu_{n}=4^{n}
$$

violating inequality (2).

Theorem 5. Let $p \in \mathbb{R}, \beta:(0, \infty) \rightarrow \mathbb{R}$ and let $p>1$. Then there exists a sequence $f_{n}$ and a function $\beta$ satisfying the conditions $\beta_{0}<1-(1 / p), \beta_{\infty}<1-(1 / p)$, and

$$
\lim _{n \rightarrow \infty}\left|\beta\left(\delta_{n}\right)-\beta_{0}\right| \ln \frac{1}{\delta_{n}}=\infty: \delta_{n}=4^{-n}
$$

or

$$
\lim _{n \rightarrow \infty}\left|\beta\left(\mu_{n}\right)-\beta_{\infty}\right| \ln \mu_{n}=\infty: \mu_{n}=4^{n}
$$

violating inequality (2).

\section{Proof of Main Results}

For the proof of Theorems 1 and 2 we refer to [8]. Other proofs of these theorems are given in [10]. The proof of Theorem 3 also is given in [8]. Here we derive an alternative proof of that theorem using the general results of $[2,4]$.

In the proof of main results we use the following elementary Lemma.

Lemma 6. Suppose $s: \mathbb{R} \rightarrow(0, \infty)$ is a measurable function such that $s \in \Lambda_{0} \cap \Lambda_{\infty}$ and $0<s^{-}, s^{+}<\infty$; then it holds the estimate

$$
C_{1}^{-1} x^{s(0)} \leq x^{s(x)} \leq C_{1} x^{s(0)},
$$

for $0<x<1$ and the estimate

$$
C_{2}^{-1} x^{s(\infty)} \leq x^{s(\infty)} \leq C_{2} x^{s(\infty)}
$$

for $x \geq 2$.

Proof of Theorem 3. To prove Theorem 3 we apply the results of $[2,4]$, where it was proved that the following conditions

$$
\begin{gathered}
\sup _{x \in(0,1)} V(x)^{1 / q(0)} W(x)^{((p(0)-1) / p(0))}<\infty, \\
\sup _{x \in(2, \infty)} V(x)^{1 / q(\infty)} W(x)^{((p(\infty)-1) / p(\infty))}<\infty
\end{gathered}
$$

are necessary and sufficient for inequality (1) to hold if $q(0) \geq$ $p(0), q(\infty) \geq p(\infty)$, and the regularity conditions are satisfied:

$$
\begin{aligned}
& \limsup _{x \rightarrow 0}[p(x)-p(0)] \ln \frac{1}{W(x)}<\infty ; \\
& \limsup _{x \rightarrow \infty}[p(x)-p(\infty)] \ln \frac{1}{V(x)}<\infty,
\end{aligned}
$$

where $V(x)=\int_{x}^{\infty} v(t) d t, W(x)=\int_{0}^{x} \omega^{-1 /(p(t)-1)} d t$.

In Theorem 3, we have accepted that $q(x)=p(x)$ and $V(x):=V_{1}(x)=\int_{x}^{\infty} t^{(\beta(t)-1) p(t)} d t, W(x):=W_{1}(x)=$ $\int_{0}^{x} t^{-(\beta(t) p(t)) /(p(t)-1)} d t$. It is easy to show that the conditions $p, \beta \in \Lambda_{\infty}$ imply $(\beta-1) p \in \Lambda_{\infty}$. Therefore, it follows from Lemma 6 that

$$
C_{3}^{-1} x^{(\beta(\infty)-1) p(\infty)} \leq x^{(\beta(x)-1) p(x)} \leq C_{3} x^{(\beta(\infty)-1) p(\infty)}
$$

for $x \geq 2$ by some $C_{3}>0$. Also the conditions $p, \beta \in \Lambda_{0}$ imply $-(\beta p /(p-1)) \in \lambda_{0}$. Therefore, it follows from Lemma 6 that

$$
\begin{aligned}
C_{4}^{-1} x^{-(\beta(0) p(0) /(p(0)-1))} & \leq x^{-(\beta(x) p(x) /(p(x)-1))} \\
& \leq C_{4} x^{-(\beta(0) p(0) /(p(0)-1))}
\end{aligned}
$$


for $0<x<1$ by some $C_{4}>0$. Integrating these inequalities over the intervals $(x, \infty)$ and $(0, x)$, respectively, we get

$$
\begin{aligned}
C_{5}^{-1} x^{(\beta(\infty)-1) p(\infty)+1} \leq & V_{1}(x) \leq C_{5} x^{(\beta(\infty)-1) p(\infty)+1}, \quad x>2, \\
C_{6}^{-1} x^{1-(\beta(0) p(0) /(p(0)-1))} & \leq W_{1}(x) \\
& \leq C_{6} x^{1-(\beta(0) p(0) /(p(0)-1))}, \quad 0<x<1 .
\end{aligned}
$$

To complete the proof of Theorem 3 it suffices to apply estimates (20) to verify conditions (16) and (17). Now, Theorem 3 follows from the upper refereed results of the works $[2,4]$.

Proof of Lemma 6. Let $0<x \leq \delta$ and let $s(x) \geq s(0)$; then we have $x^{s(x)-s(0)} \leq 1+\delta^{s^{+}-s(0)}$, where $\delta$ is a certain number from the interval $(0,1)$. If $0<x \leq \delta$ and $s(x)<s(0)$ then by condition $s \in \Lambda_{0}$ we have

$$
\begin{aligned}
x^{s(x)-s(0)} & =\left(\frac{\delta}{x}\right)^{s(0)-s(x)} \delta^{s(x)-s(0)} \\
& \leq\left(\frac{\delta}{x}\right)^{c_{1} / \ln (1 / x)} \delta^{s(x)-s(0)} \\
& =e^{c_{1}} \delta^{s(x)-s(0)+c_{1} / \ln (1 / x)} \\
& \leq e^{c_{1}}\left(1+\delta^{c_{1} / \ln (1 / \delta)}\right)\left(1+\left(\frac{1}{\delta}\right)^{s(0)-s^{-}}\right) .
\end{aligned}
$$

Therefore, for $0<x \leq \delta$ we have the estimation

$$
x^{s(x)-s(0)} \leq C,
$$

where the positive constant $C$ depends on $s^{-}, s^{+}, s(0), \delta$. Same inequality holds for the function $x^{s(0)-s(x)}$. Indeed, for $0<x \leq$ $\delta$ and $s(x) \geq s(0)$ we have $x^{s(0)-s(x)} \leq 1+\delta^{s(0)-s^{-}}$. If $0<x \leq \delta$ and $s(x) \geq s(0)$ by condition $s \in \Lambda_{0}$ we have

$$
\begin{aligned}
x^{s(0)-s(x)} & =\left(\frac{\delta}{x}\right)^{s(x)-s(0)} \delta^{s(0)-s(x)} \\
& \leq\left(\frac{\delta}{x}\right)^{c_{1} / \ln (1 / x)} \delta^{s(0)-s(x)} \\
& =e^{c_{1}} \delta^{s(0)-s(x)+c_{1} \ln (1 / x)} \\
& \leq e^{c_{1}}\left(1+\delta^{c_{1} \ln (1 / x)}\right)\left(1+\left(\frac{1}{\delta}\right)^{s^{+}-s(0)}\right) .
\end{aligned}
$$

By using these inequalities and by the representation

$$
x^{s(x)}=x^{s(0)} x^{s(x)-s(0)}
$$

we have estimate (14).

To show estimate (15) note that for $x \geq M$ and $s(x) \leq$ $s(\infty)$ we have $x^{s(x)-s(\infty)} \leq 1+(1 / M)^{s(\infty)-s^{-}}$, where the $M \geq 2$ is a certain number. If $x \geq M$ and $s(x) \geq s(\infty)$ then by the condition $s \in \Lambda_{\infty}$ we have

$$
\begin{aligned}
x^{s(x)-s(\infty)} & =\left(\frac{x}{M}\right)^{s(x)-s(\infty)} M^{s(x)-s(\infty)} \\
& \leq\left(\frac{x}{M}\right)^{C_{2} / \ln x} M^{s(x)-s(\infty)} \\
& =e^{C_{2}} M^{s(x)-s(\infty)-C_{2} / \ln x} \\
& \leq e^{C_{2}}\left(1+\left(\frac{1}{M}\right)^{C_{2} / \ln M}\right)\left(1+M^{s(\infty)-s^{+}}\right) .
\end{aligned}
$$

Combining the estimates for the functions $x^{s(x)-s(\infty)}$, $x^{s(\infty)-s(x)}$ for $x \geq M$ by the presentation

$$
x^{s(x)}=x^{s(\infty)} x^{s(x)-s(\infty)}
$$

we get estimate (15). To complete the proof of Lemma 6, note that the condition $s \in \Lambda_{0}$ is equivalent to

$$
-C \leq|s(x)-s(0)| \ln \frac{1}{x} \leq C
$$

and the $s \in \Lambda_{\infty}$ is equivalent to

$$
-C \leq|s(x)-s(\infty)| \ln x \leq C,
$$

respectively.

Proof of Theorem 4. Let us assume that $f_{k}(x)=$ $x^{-(1 / \beta(x))-\beta} \chi_{\left(\delta_{k}, 2 \delta_{k}\right)}(x) ; x \in(0, \infty)$. Fix $k \in \mathbb{N}$. We define the step function

$$
p(x)=\left\{\begin{array}{ll}
p_{0}+a_{n} & \text { if } x \in\left(2 \delta_{n}, 4 \delta_{n}\right) \\
p_{0} & \text { if } x \in\left(\delta_{n}, 2 \delta_{n}\right),
\end{array} \quad n \in \mathbb{N} .\right.
$$

Here $\alpha_{n}$ is a sequence of positive numbers that satisfies the condition $n \alpha_{n} \rightarrow \infty$ as $n \rightarrow \infty$. Then $a_{n} \cdot \ln \left(1 / \delta_{n}\right) \rightarrow \infty$ as $n \rightarrow \infty$ and condition (10) is fulfilled for the function $p(x)$. We have

$$
\begin{gathered}
I_{p(\cdot)}\left(x^{\beta(x)} \cdot f_{k}(x)\right)=\int_{\delta_{n}}^{2 \delta_{n}}\left(t^{\beta} \cdot t^{-\left(1 / p_{0}\right)-\beta}\right)^{p_{0}} d t=\ln 2<\infty, \\
I_{p(\cdot)}\left(x^{\beta(x)-1} \cdot H f_{k}(x)\right) \\
\geq \int_{2 \delta_{n}}^{4 \delta_{n}}\left[\int_{\delta_{n}}^{2 \delta_{n}} t^{-(1 / p(t))-\beta} d t\right]^{p_{0}+\alpha_{n}} \cdot x^{\left(\beta_{0}-1\right) \cdot\left(p_{0}+\alpha_{n}\right)} d x \\
\geq \int_{2 \delta_{n}}^{4 \delta_{n}} \delta_{n}^{\left(1-\left(1 / p_{0}\right)-\beta\right)\left(p_{0}+\alpha_{n}\right)} \cdot x^{(\beta-1) \cdot\left(p_{0}+\alpha_{n}\right)} d x \\
\geq C \cdot \delta_{n}^{-\alpha_{n} / p_{0}}=C \cdot e^{\alpha_{n} / p_{0} \ln \left(1 / \delta_{n}\right)} \longrightarrow \infty
\end{gathered}
$$

as $n \rightarrow \infty$. The last relation shows violating of inequality (2) for sufficiently large $n$. 
We define $f_{k}(x)=x^{-(1 / p(x))-\beta} \chi_{\left(\mu_{k}, 2 \mu_{k}\right)}(x)$ for $x \in(0, \infty)$. Fix $k \in \mathbb{N}$. We also define the step function

$$
p(x)=\left\{\begin{array}{ll}
p_{\infty}-a_{n} & \text { if } x \in\left(2 \mu_{n}, 4 \mu_{n}\right) \\
p_{\infty} & \text { if } x \in\left(\mu_{n}, 2 \mu_{n}\right),
\end{array} \quad n \in \mathbb{N},\right.
$$

where $n \alpha_{n} \rightarrow \infty$ as $n \rightarrow \infty$. We have $\alpha_{n} \ln \mu_{n} \rightarrow \infty$ as $n \rightarrow \infty$ and condition (11) holds for the function $p(x)$. Furthermore,

$$
\begin{gathered}
I_{p(\cdot)}\left(x^{\beta} \cdot f_{k}(x)\right)=\int_{\mu_{n}}^{2 \mu_{n}}\left(x^{\beta} \cdot x^{-\left(1 / p_{\infty}\right)-\beta}\right)^{p_{\infty}} d x=\ln 2<\infty, \\
I_{p(\cdot)}\left(x^{\beta-1} \cdot H f_{k}(x)\right) \\
\geq \int_{2 \mu_{n}}^{4 \mu_{n}}\left[\int_{\mu_{n}}^{2 \mu_{n}} t^{-(1 / p(t))-\beta} d t\right]^{\left(p_{\infty}-\alpha_{n}\right)} \cdot x^{(\beta-1)\left(p_{\infty}-\alpha_{n}\right)} d x \\
\geq C \mu_{n}^{\alpha_{n} / p_{\infty}}=C e^{\left(\alpha_{n} / p_{\infty}\right) \ln \mu_{n}} \longrightarrow \infty
\end{gathered}
$$

as $n \rightarrow \infty$, which contradicts (2) for sufficiently large $n$.

Proof of Theorem 5. Let us assume that $f_{k}(x)=$ $x^{-(1 / p)-\beta(x)} \chi_{\left(\delta_{k}, 2 \delta_{k}\right)}(x) ; x \in(0, \infty)$. Fix $k \in \mathbb{N}$. We define the step function $\beta$ as

$$
\beta(x)=\left\{\begin{array}{ll}
\beta_{0}+a_{n} & \text { if } x \in\left(\delta_{n}, 2 \delta_{n}\right) \\
\beta_{0} & \text { if } x \in\left(2 \delta_{n}, 4 \delta_{n}\right),
\end{array} \quad n \in \mathbb{N},\right.
$$

where $a_{n} \cdot \ln \left(1 / \delta_{n}\right) \rightarrow \infty$. Then,

$$
\begin{aligned}
& I_{p(\cdot)}(\left.x^{\beta(x)} \cdot f_{k}(x)\right) \\
&=\int_{\delta_{n}}^{2 \delta_{n}}\left(x^{\beta_{0}+\alpha_{n}} \cdot x^{-(1 / p)-\beta_{0}-\alpha_{n}}\right)^{p} d x \\
&=\ln 2<\infty, \\
& I_{p(\cdot)}\left(x^{\beta(x)-1} \cdot H\left(f_{k}(x)\right)\right) \\
& \quad \geq \int_{2 \delta_{n}}^{4 \delta_{n}}\left[\int_{\delta_{n}}^{2 \delta_{n}} t^{-(1 / p)-\beta_{0}-\alpha_{n}} d t\right]^{p} \cdot x^{\left(\beta_{0}-1\right) p} d x \\
& \quad=C \cdot \delta_{n}^{-p \alpha_{n}}=C \cdot e^{p \alpha_{n} \ln \left(1 / \delta_{n}\right)} \longrightarrow \infty
\end{aligned}
$$

as $n \rightarrow \infty$. The last relation contradicts the validity of inequality (2).

We define $f_{k}(x)=x^{-(1 / x)-\beta(x)} \chi_{\left(\mu_{k}, 2 \mu_{k}\right)}(x) ; x \in(0, \infty)$. Fix $k \in \mathbb{N}$, where the function $\beta$ is defined as

$$
\beta(x)=\left\{\begin{array}{ll}
\beta_{\infty}-a_{n} & \text { if } x \in\left(\mu_{n}, 2 \mu_{n}\right) \\
\beta_{\infty} & \text { if } x \in\left(2 \mu_{n}, 4 \mu_{n}\right),
\end{array} \quad n \in \mathbb{N},\right.
$$

where $\lim _{n \rightarrow \infty} a_{n} \cdot \ln \mu_{n}=\infty$. Then,

$$
\begin{aligned}
& I_{p(\cdot)}(\left.x^{\beta(x)} \cdot f_{k}(x)\right) \\
&=\int_{\mu_{n}}^{2 \mu_{n}}\left(x^{\beta_{\infty}-\alpha_{n}} \cdot x^{-(1 / p)-\beta_{\infty}+\alpha_{n}}\right)^{p} d x \\
&=\ln 2<\infty, \\
& I_{p(\cdot)}\left(x^{\beta(x)-1} \cdot H\left(f_{k}(x)\right)\right) \\
& \quad \geq \int_{2 \mu_{n}}^{4 \mu_{n}}\left[\int_{\mu_{n}}^{2 \mu_{n}} t^{-(1 / p)-\beta_{\infty}+\alpha_{n}} d t\right]^{p} \cdot x^{\left(\beta_{\infty}-1\right) p} d x \\
& \quad \geq \int_{2 \mu_{n}}^{3 \mu_{n}} \mu_{n}^{\left(1-(1 / p)-\beta_{\infty}+\alpha_{n}\right) p} \cdot x^{\left(\beta_{\infty}-1\right) p} d x \\
& \geq C \cdot \mu_{n}^{p \alpha_{n}}=C \cdot e^{p \alpha_{n} \ln \mu} \longrightarrow \infty
\end{aligned}
$$

as $n \rightarrow \infty$ which contradicts inequality (2).

\section{Conflict of Interests}

The authors declare that they have no conflict of interests regarding the publication of this paper.

\section{Acknowledgment}

The authors are grateful to the anonymous referee for carefully reading the paper and making useful comments.

\section{References}

[1] F. I. Mamedov and A. Harman, "On a weighted inequality of Hardy type in spaces $L^{p(\cdot)}$," Journal of Mathematical Analysis and Applications, vol. 353, no. 2, pp. 521-530, 2009.

[2] F. I. Mamedov and Y. Zeren, "On equivalent conditions for the general weighted Hardy type inequality in space $L^{p(\cdot)}$," Zeitschrift für Analysis und ihre Anwendungen, vol. 31, no. 1, pp. 55-74, 2012.

[3] F. I. Mamedov and A. Harman, "On a Hardy type general

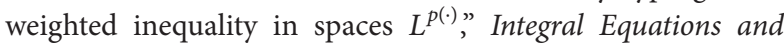
Operator Theory, vol. 66, no. 4, pp. 565-592, 2010.

[4] D. Cruz-Uribe and F. I. Mamedov, "On a general weighted Hardy type inequality in the variable exponent Lebesgue spaces," Revista Matemática Complutense, vol. 25, no. 2, pp.335367, 2012.

[5] L. Diening and S. Samko, "Hardy inequality in variable exponent Lebesgue spaces," Fractional Calculus \& Applied Analysis, vol. 10, no. 1, pp. 1-18, 2007.

[6] P. Harjulehto, P. Hästö, and M. Koskenoja, "Hardy's inequality in a variable exponent Sobolev space," Georgian Mathematical Journal, vol. 12, no. 3, pp. 431-442, 2005.

[7] A. Harman and F. I. Mamedov, "On boundedness of weighted Hardy operator in $L^{p(\cdot)}$ and regularity condition," Journal of Inequalities and Applications, vol. 2010, Article ID 837951, 14 pages, 2010.

[8] A. Harman, "On necessary condition for the variable exponent Hardy inequality," Journal of Function Spaces and Applications, vol. 2012, Article ID 385925, 6 pages, 2012. 
[9] R. Mashiyev, B. Çekiç, F. I. Mamedov, and S. Ogras, "Hardy's inequality in power-type weighted $L^{p(\cdot)}(0, \infty)$ spaces," Journal of Mathematical Analysis and Applications, vol. 334, no. 1, pp. 289-298, 2007.

[10] F. I. Mamedov, "On Hardy type inequality in variable exponent Lebesgue space $L^{p(\cdot)}(0, l)$," Azerbaijan Journal of Mathematics, vol. 2, no. 1, pp. 90-362, 2012.

[11] A. Kufner and L.-E. Persson, Weighted Inequalities of Hardy Type, World Scientific, River Edge, NJ, USA, 2003.

[12] O. Kováčik and J. Rákosník, "On spaces $L^{p(x)}$ and $W^{k, p(x), "}$ Czechoslovak Mathematical Journal, vol. 41, no. 116, pp. 592-618, 1991. 


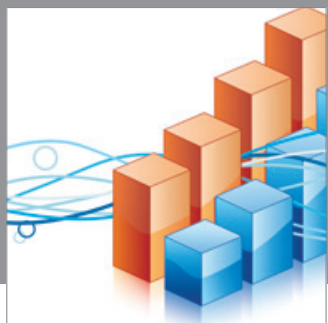

Advances in

Operations Research

mansans

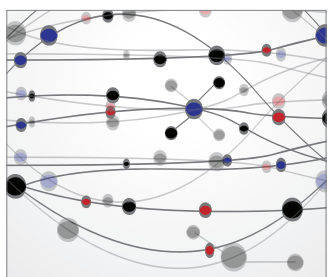

The Scientific World Journal
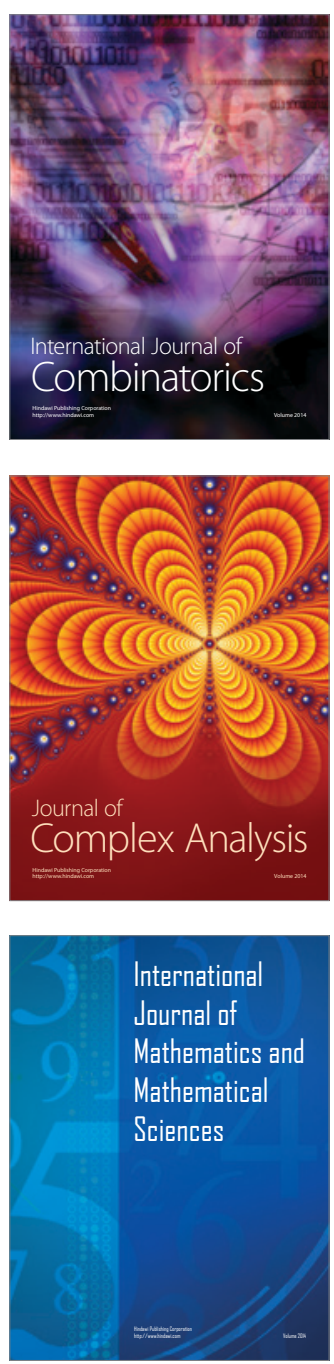
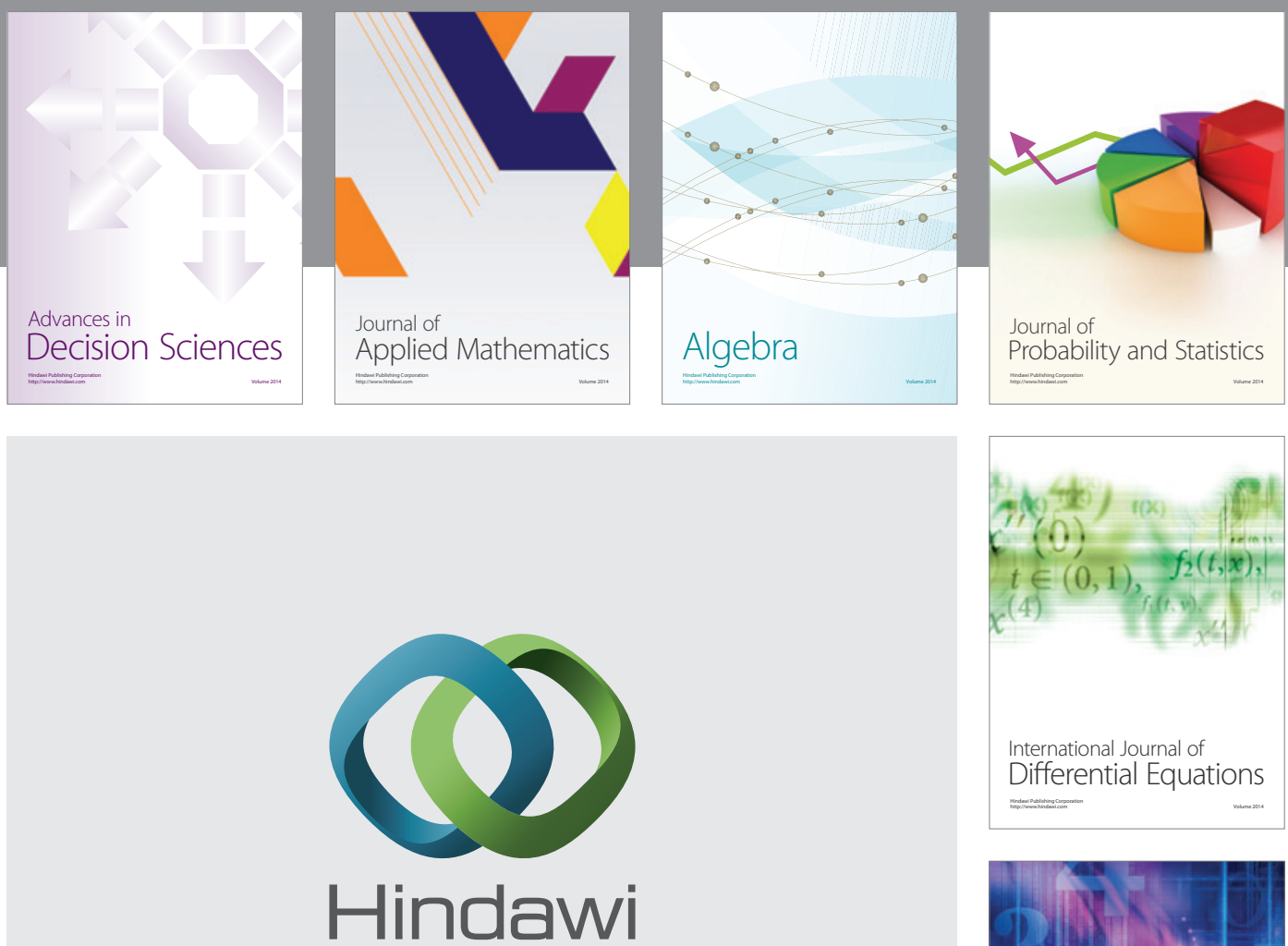

Submit your manuscripts at http://www.hindawi.com
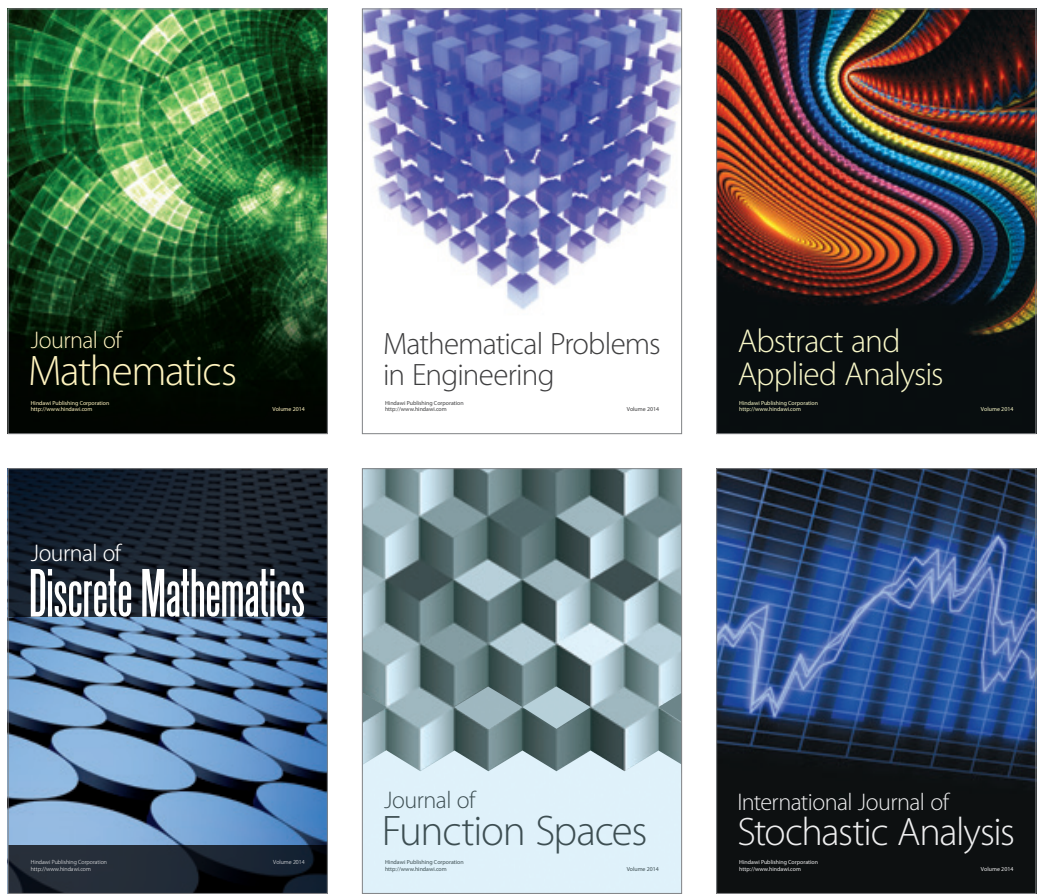

Journal of

Function Spaces

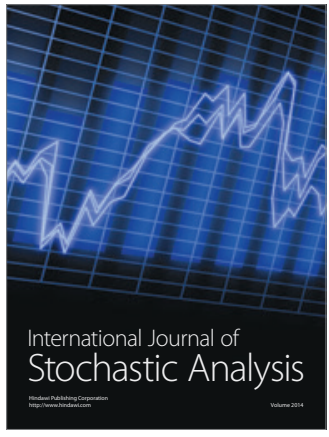

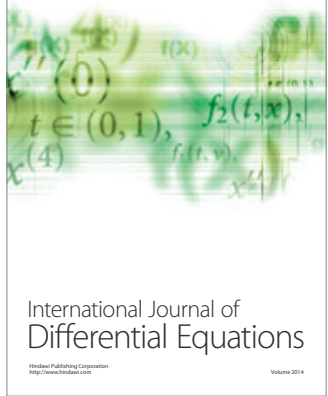
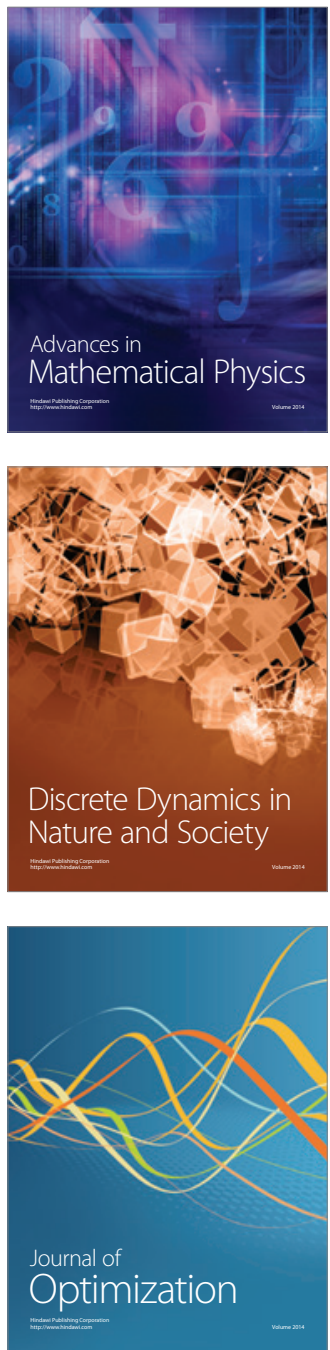